\title{
CITTADINANZA DIFFERENZIATA \\ E INTEGRAZIONE MULTICULTURALE
}

\author{
di Matteo Gianni
}

Dopo un lungo periodo di disinteresse, i teorici della politica ricominciano a considerare seriamente il ruolo che le minoranze culturali esercitano sul funzionamento e la legittimità normativa dei sistemi democratici. Le società liberali sono caratterizzate da tensioni politiche e sociali profonde, che rischiano di paralizzarne il funzionamento e di rimetterne in causa la stabilità. Come afferma un eminente filosofo liberale «dar risposta a tali questioni costituisce probabilmente la sfida più grande che oggi le democrazie si trovano a fronteggiare» (Kymlicka 1995b, 1). Se tutti concordano sull'idea che il problema è serio, le soluzioni proposte sono molto diverse. Ciò non deve sorprendere visto che $\mathrm{i}$ rimedi avanzati dipendono in larga misura dalla maniera in cui il problema da risolvere viene interpretato e costruito analiticamente.

Essendo estremamente cariche di significati ideologici e politici, nozioni come multiculturalismo e integrazione sono difficilmente costruibili in modo neutro e obiettivo. Fondamentalmente, esse sono impiegate in due accezioni diverse - normativa e descrittiva - fatto che spesso crea confusione e non facilita la possibilità di trovare delle soluzioni consensuali fra le varie correnti teoriche e politiche. Il multiculturalismo, in effetti, può essere inteso sia come un fatto sociale - caratterizzato dalla presen$\mathrm{za}$, in un dato contesto politico, di gruppi che rivendicano delle identità culturali diverse (e spesso antagoniste) - che come un insieme di politiche pubbliche atte a favorire la convivenza politica e sociale delle varie minoranze culturali. In questo senso, il multiculturalismo è visto come un valore che bisogna difendere $\mathrm{e}$ promuovere. In fondo, la valenza politica della relazione fra multiculturalismo e integrazione risiede precisamente nell'articolazione fra il discorso prescrittivo (quale dovrebbe essere la migliore concezione dell'integrazione nell'ambito di una società

RIVISTA ITALIANA DI SCIENZA POLITICA / a. XXVII, n. 3, dicembre 1997 
multiculturale?) e l'analisi empirica (quali sono le modalità d'integrazione in seno alle società multiculturali?). I due tipi di riflessione si influenzano reciprocamente: il «come» nutre il «dovrebbe» e il «dovrebbe» stabilisce i criteri di misura del «come».

In questo articolo proporrò una possibile interpretazione della relazione teorica fra multiculturalismo e integrazione politica. Vorrei mostrare che la tematica dei legami fra multiculturalismo e democrazia è più ampia delle analisi dei flussi migratori, dei rapporti fra comunità etnolinguistiche o delle relazioni fra Stato e comunità religiose sulle quali si focalizza gran parte della ricerca in Europa. Da un punto di vista teorico, questi aspetti mi sembrano costituire le dimensioni di uno stesso fenomeno più generale. Si tratta della «crisi» della cittadinanza come principio organizzatore dell'identità politica (Beiner 1995; Gianni 1995) e, dunque, della democrazia rappresentativa. In quest'ottica, ad esempio, le tensioni legate all'immigrazione (e la paranoia politica che essa solleva nelle democrazie liberali industrializzate) sono da ritenersi più un rivelatore del problema generale dell'integrazione multiculturale che non la sua stessa causa. Le società multiculturali sono percorse da una dialettica costante fra dinamiche d'integrazione e dinamiche di differenziazione sociale, politica e culturale. Se la differenziazione prevale sull'integrazione, le possibilità di raggiungere un accordo generale sulle regole del gioco democratico e delle politiche che ne scaturiscono diminuiscono drasticamente. Siamo chiari: un certo grado di conflitto sociale e politico è endemico a ogni società liberale. Su questo, praticamente tutti i teorici della democrazia, al di là delle loro differenze paradigmatiche, concordano. $\mathrm{Ma}$ i conflitti culturali hanno caratteristiche diverse. Non sono percepiti come un elemento dinamico e immanente del gioco democratico, ma dei potenziali fattori di distruzione e blocco di quest'ultimo. Un semplice sguardo alla situazione nell'ex-Jugoslavia e ai conflitti etno-nazionalistici nell'Europa dell'Est non può che confermare questa percezione.

Il fatto sociale del multiculturalismo, quindi, impone un ripensamento dei presupposti normativi che legittimano la democrazia liberale. Più specificamente, si tratta di riconsiderare il potenziale normativo e politico della cittadinanza liberale come fattore d'integrazione politica nel quadro delle società multiculturali. In quest'articolo presenterò qualche elemento di riflessione in materia. Difenderò la tesi secondo la quale la nozione di cittadinanza differenziata (Kymlicka 1995a; Young 1990) apre 
delle prospettive analitiche interessanti per concepire delle migliori modalità d'integrazione politica nel quadro di società traversate da conflitti culturali profondi. Un tale presupposto implica una critica piuttosto radicale della concezione liberale classica della cittadinanza e del modello d'integrazione che gli è soggiacente. Secondo questo approccio, infatti, l'attribuzione della cittadinanza rappresenta il vettore principale dell'integrazione politica. Essa costituisce il comun denominatore che permette di unire, in una stessa sfera politico-giuridica, degli individui divisi nei loro stili di vita e nelle loro appartenenze culturali. Detto con altre parole, la cittadinanza liberale si basa sul principio di non-discriminazione pubblica (o di neutralità) nei confronti delle diverse identità culturali. Come riassume efficacemente Amy Gutmann:

la nostra non identificazione con le istituzioni del servizio pubblico, ovvero l'impersonalità delle istituzioni pubbliche, è il prezzo che i cittadini devono essere disposti a pagare per vivere in una società che li tratti tutti da uguali indipendentemente dalla loro identità particolare, etnica, religiosa, razziale o sessuale $(1993,13)$.

La concezione della cittadinanza differenziata implica delle modifiche sostanziali di questo modello normativo. Il riconoscimento delle differenze presuppone infatti che le istituzioni politiche non siano «cieche» nei confronti delle specificità culturali ma che, invece, le prendano, seriamente, in considerazione. La nozione di riconoscimento pubblico delle identità culturali acquisisce dunque un'importanza normativa fondamentale, che implica una maniera diversa d'impostare le varie politiche pubbliche in materia d'integrazione. In questo contributo non discuterò la natura e le modalità di produzione di queste ultime. Il mio intento è semplicemente quello di fornire qualche indicazione valutativa. Ė questo, ritengo, lo scopo della teoria politica: valutare, criticare e orientare il senso e i fondamenti delle pratiche politiche.

Il fatto di considerare analiticamente seducente l'idea di un riconoscimento pubblico delle differenze culturali non deve far dimenticare che questa nozione solleva una serie di questioni teoriche importanti ${ }^{1}$. Come sostiene Taylor $(1993,59)$, la politi-

1 Secondo Lukes «il riconoscimento delle identità collettive è un concetto complesso ed ancora insufficientemente analizzato, che solleva vari problemi fondamentali» (1995, 1). 
ca del riconoscimento «ci chiede di concedere un riconoscimento e uno status a qualcosa di non condiviso universalmente». Questo non è un aspetto da sottovalutare, visto che le lotte sociali e politiche per affermare la cittadinanza sono state fatte in nome della distruzione dei privilegi delle categorie dominanti (si tratti degli Ordini nell'ancien régime o del dominio della borghesia). Dunque, la politica del riconoscimento sembrerebbe costituire un «passo indietro» rispetto alle conquiste civili, politiche e sociali della cittadinanza. In altri termini, comporterebbe la distruzione del fondamento universalista della cittadinanza in nome d'interessi e identità particolaristiche, non condivisibili da tutti gli attori politici. Il riconoscimento, quindi, implicherebbe la differenziazione (o addirittura la disintegrazione) dello spazio pubblico e condurrebbe, ineluttabilmente, all'esacerbazione del conflitto sociale, visto che distruggerebbe l'identità politica minima dei cittadini che legittima e sostiene il funzionamento delle istituzioni democratiche.

Benché pertinenti, queste critiche mi sembrano troppo radicali e basate su un eccesso d'ottimismo al riguardo del potenziale integrativo del modello liberale. A mio modo di vedere, certe forme di riconoscimento sono necessarie per ottenere una migliore integrazione politica e sociale nell'ambito di una società culturalmente differenziata, nella quale i membri di certi gruppi godono di una cittadinanza di «secondo grado» a causa delle loro appartenenze culturali. Contrariamente ai suoi detrattori, credo che il riconoscimento, se ben concepito, può condurre ad un rafforzamento della cittadinanza come vettore d'integrazione politica.

\section{Concezioni del multiculturalismo}

Il riconoscimento pubblico non è una mera categoria giuridica. Nelle società multiculturali liberali il riconoscimento rappresenta una risorsa politica rara, per la cui acquisizione e controllo della quale nascono dei conflitti fra gli attori politici ${ }^{2}$. Questo tipo di conflitti verte sulla rivendicazione, da parte di certi gruppi minoritari, del riconoscimento pubblico delle loro identità culturali (Gianni 1995). Ė per questo che:

2 La mia interpretazione del concetto di conflitti di riconoscimento si ispira a Pizzorno (1993). 
il problema di come e se i gruppi culturali debbano ricevere un riconoscimento politico è uno dei più acuti e tormentati fra quelli che molte società democratiche e democratizzanti trovano davanti a sé oggi (Gutmann 1993, 14).

Una parte importante del problema risiede nella difficoltà di definire cos'è un gruppo culturale, ossia il $c h i$ deve beneficiare del riconoscimento pubblico. In questo senso, è interessante constatare le differenze di percezione del problema del multiculturalismo in Europa e negli Stati Uniti. In generale, in Europa si parla di multiculturalismo quando gruppi religiosi, etnici o linguistici differenti devono convivere nello stesso sistema politico. La riflessione verte quindi essenzialmente su due problematiche: quella della gestione del rapporto fra minoranze etnolinguistiche (pensiamo alle ricerche concernenti il federalismo o il nazionalismo) e quella dell'integrazione culturale delle comunità d'immigrati e rifugiati che affluiscono nelle democrazie in cerca di benessere o di protezione politica. Nell'ambito nordamericano, invece, la nozione di multiculturalismo esprime una realtà sociale più vasta. Oltre alle minoranze razziali, etniche, e religiose, altri gruppi come le donne, le minoranze sessuali o gli handicappati rientrano in questa problematica generale. Sostanzialmente, il multiculturalismo esprime le relazioni fra $\mathrm{i}$ «diversi» e i «normali», fra la/e maggioranza/e culturalmente (e politicamente) dominante/i e le categorie escluse o marginalizzate. Più che sul termine cultura, l'attenzione si focalizza sui concetti d'identità e di differenza (Connolly 1991). Vi sono delle ragioni storiche e politiche che spiegano questo tipo di costruzione della nozione. Il fallimento della politica del melting pot ha rivelato la forza dei processi di esclusione razziale ed etnica che hanno marcato la costruzione dell' «associazione di cittadini» americana (Walzer 1992). Inoltre, il soggettivismo politico degli anni ' 60 ha trasformato il tema delle identità culturali e della loro integrazione in un problema politico cruciale. Gitlin (1995) non esita a utilizzare l'espressione «guerra delle culture» per descrivere il tipo di dinamica socio-politica che ne è risultata. La nonterritorializzazione delle comunità culturali (salvo qualche eccezione) e la storia di oppressioni e esclusioni su basi culturali fanno sì che la problematica del multiculturalismo sia percepita in modo differente rispetto a società nelle quali le differenze culturali sono territorializzate (come nel caso dei sistemi federalisti) o per le quali la finzione dello Stato nazionale costituisce un collante simbolico ancora potente. 
Nonostante ciò, al di là dell'influenza del contesto, il problema teorico della definizione di $\cos ^{\prime} \hat{e}$ un gruppo culturale resta fondamentale. In effetti, a seconda della definizione adottata, il numero e le caratteristiche intrinseche dei gruppi incorporati nella problematica del multiculturalismo saranno molto differenti. E possibile considerare le donne o gli handicappati come un gruppo culturale alla stessa stregua dei gruppi etnici o religiosi? $\mathrm{O}$ non sarebbe meglio considerare i gruppi del primo tipo come delle classi sociali, escludendo quindi la loro dimensione culturale? ? Potrà sembrare sorprendente, ma, per la mia argomentazione, queste distinzioni non sono molto rilevanti. Invece di adottare una definizione sostanziale del concetto di cultura, ritengo più utile ricorrere a un approccio pragmatico, teso non tanto a determinare se un gruppo merita o no l'etichetta di «gruppo culturale», ma ad analizzare quello che è fatto politicamente nel nome di un'identità culturale ${ }^{4}$. Un esempio può aiutare a capire questo punto. Prendiamo la situazione italiana negli ultimi tempi. Il dibattito pubblico è stato marcato - fra l'altro - da tre temi in un modo o nell'altro legati a rivendicazioni fondate su una concezione dell'identità culturale. Mi riferisco al discorso federalista della Lega Nord, al problema dell'accesso e dell'integrazione delle varie comunità immigrate (soprattutto extracomunitarie), e alla normativa che ha trasformato lo stupro femminile da reato contro la morale a reato contro la persona. Sicuramente, per l'antropologo e per il sociologo, esistono differenze fondamentali circa la natura dei gruppi implicati. Ma per il politologo, che deve analizzare le cause e gli effetti politici dei loro atti, è possibile reperire una sorta di comune denominatore: la richiesta di riconoscimento pubblico della loro (presunta) discriminazione culturale; il carattere iniquo del trattamento ricevuto dalle istituzioni politiche. $\mathrm{Al}$ di là delle loro differenze, quindi, le rivendicazioni che ne sono conseguite esprimono una preoccupazione analoga: l'esigenza di una migliore approssimazione politica degli ideali incarnati dalla cittadinanza democratica.

$\mathrm{Al}$ di là dei problemi teorici che solleva, la concezione «lar-

3 Questo suggerimento teorico mi è stato dato da Michael Walzer, che tengo qui a ringraziare.

4 Devo quest'idea a Gellner, secondo il quale «forse è preferibile affrontare il concetto di cultura senza cercare troppo di avviarsi verso una definizione formale, ma esaminando piuttosto ciò che è prodotto dalla cultura» $(1989,19)$. 
ga» del multiculturalismo, ispirata al modello nord-americano, permette dunque di cogliere un aspetto importante delle tensioni che attraversano le democrazie liberali: la messa in causa della cittadinanza come principio costitutivo dell'identità politica e, quindi, come fattore d'integrazione dei vari attori sociali nello spazio pubblico. Il problema del multiculturalismo non investe solo il modo di integrare coloro che non dispongono della cittadinanza e desiderano ottenerla, ma anche coloro che, pur possedendola, non si riconoscono nei valori culturali e nel tipo d'identità espressi da questo status formale e nello Stato che lo attribuisce. Mi sembra utile distinguere quattro possibilità teoriche d'atteggiamento collettivo al riguardo della cittadinanza: $i$ ) i gruppi di cittadini integrati, che riconoscono e considerano legittimi i valori espressi dalla cittadinanza; ii) i gruppi di cittadini differenti che, per varie ragioni, non si riconoscono e contestano i valori espressi dalla cittadinanza; iii) i gruppi di non cittadini integrati, che, malgrado non dispongano della cittadinanza, perseguono una strategia d'integrazione nella nuova comunità; iv) i gruppi di non cittadini non integrati che, per scelta o a causa delle difficoltà incontrate nella loro integrazione, difendono una strategia comunitaria e «differenzialista». L'azione di questi quattro gruppi di attori - basata sulla tensione fra integrazione e differenziazione o, usando la terminologia di Hirschman, fra exit, voice e loyalty - rende ardua l'impresa di definire una teoria della cittadinanza adatta al problema del multiculturalismo $\mathrm{e}$, in modo più generale, di proporre una soluzione normativa univoca al problema dell'integrazione politica. Benché non esista un consenso sui possibili rimedi, gli autori sono relativamente d'accordo nel denunciare una delle conseguenze più perniciose della democrazia: la mancata integrazione di un numero sempre maggiore di attori politici (soprattutto se dettata da ragioni strutturali) rimette in causa il senso stesso dell'ideale democratico e rappresenta un pericolo per l'ordine sociale (Taylor 1993; Rawls 1993).

Malgrado il suo carattere generale, la tipologia appena presentata consente di osservare che - al di là dei presupposti assimilazionistici che sottendono la concezione della cittadinanza come espressione della nazionalità - l'acquisizione della cittadinanza individuale (nelle sue diverse componenti: civile, politica e sociale) non conduce necessariamente ad un'integrazione effettiva degli appartenenti alle minoranze culturali. Nell'ambito delle società multiculturali, in altre parole, l'attribuzione della 
cittadinanza costituisce una condizione necessaria, ma non sufficiente, di un'effettiva integrazione. Presa fra dinamiche contraddittorie di globalizzazione e particolarizzazione, sempre più spossessata della sua ricchezza simbolica (il che la trasforma in uno statuto essenzialmente funzionale), la cittadinanza non è più in grado di rappresentare una base comune per l'azione collettiva. In nome della cittadinanza gli attori rivendicano lealtà e forme d'appartenenza culturale che spesso sono in conflitto con i valori della cittadinanza stessa ${ }^{5}$. Come afferma Barber $(1995,3)$,

cercando una comunità identitaria, ciascuno appartiene a una tribù. Però, nessuno è cittadino, e senza cittadini, come può esserci una democrazia?

È dunque il conflitto fra tipi d'identità diversi e spesso incommensurabili che produce la tensione fra multiculturalismo e integrazione politica. Se questa diagnosi è corretta, è necessario pensare a meccanismi che siano in grado di potenziare la dimensione identitaria e integrativa della cittadinanza. Non si tratta, evidentemente, di sopprimere l'espressione delle differenze culturali (protette, nei sistemi liberali, dai principi di libertà d'espressione e di tolleranza), ma di far sì che il maggior numero possibile di attori sociali partecipi alla definizione delle regole democratiche e dei valori collettivi. È questo, infatti, il senso che attribuisco alla nozione di integrazione politica: la possibilità di disporre delle risorse politiche necessarie per poter partecipare, con ragionevoli possibilità di influenzare il risultato della deliberazione, alla determinazione dei valori collettivi. Come si è già detto, il concetto di riconoscimento apre piste teoriche interessanti per determinare modalità d'arricchimento del potenziale integrativo della cittadinanza. Il fatto che, per taluni, questi cambiamenti possano essere dedotti dal paradigma liberale stesso (Kymlicka 1995a) o, per altri, presuppongano cambiamenti sostanziali della concezione liberale (Taylor 1993) non è qui rilevante. Nella prossima sezione distinguerò due possibili interpretazioni della relazione fra cittadinanza e riconoscimento, mostrando le differenze che esse implicano in materia d'integrazione politica e di teoria della cittadinanza.

5 Per Gitlin «sono tutti i preconcetti relativi all'identità, che, con la loro assolutezza rispettiva, concorrono a dare alla questione del multiculturalismo la sua carica velenosa» $(1995,227)$. 


\section{Concezioni del riconoscimento}

Cominciamo dal significato del concetto di riconoscimento. Il riconoscimento costituisce un processo cognitivo che necessita della presenza, al minimo ${ }_{2}$ di due entità, quella che riconosce e quella che è riconosciuta. E attraverso il riconoscimento intersoggettivo che si costituisce l'identità individuale o collettiva. Essa è determinata sulla base di una serie di differenze che sono state socialmente riconosciute (Connolly 1991, 64). Una differenza non riconosciuta non è quindi visibile e perde il suo carattere di differenza.

Dal punto di vista politico, il riconoscimento può asumere forme diverse, dall'atto verbale che sancisce la visibilità politica del gruppo riconosciuto, alla protezione giuridica di quest'ultimo, fino alla determinazione di politiche positive di supporto. A seconda delle caratteristiche del sistema politico (forza politica delle minoranze, apertura o chiusura del sistema) e della sua struttura culturale (tradizioni storiche di gestione delle relazioni culturali, grado di differenziazione interna), il riconoscimento pubblico delle comunità culturali si realizzerà attraverso modalità politiche differenti. Riconoscendo l'autonomia di un gruppo etnolinguistico (come può esserlo, ad esempio, un cantone svizzero), lo Stato limita considerevolmente il suo peso politico in un dato contesto, mentre nel caso delle politiche di supporto (ad esempio l'affirmative action negli Stati Uniti) interviene fortemente nella società civile.

L'elemento che mi sembra importante sottolineare risiede nel fatto che il riconoscimento pubblico implica una trasformazione sostanziale della visibilità politica della minoranza culturale. Il gruppo, da entità culturale, diventa un'entità politica. Questo non significa che esso sia automaticamente integrato nello spazio pubblico e nelle istituzioni. Vi sono casi evidenti nei quali l'integrazione politica formale (attraverso l'attribuzione della cittadinanza) continua a essere limitata da pratiche sociali discriminatorie. Gli esempi delle minoranze afro-americana e autoctone negli Stati Uniti, o di certi gruppi etnici in Europa (come i Beurs o gli Harkis in Francia), sono in questo senso emblematici. Il modello liberale, basato sul principo di separazione fra sfera pubblica e sfera privata - che implica, di fatto, la negazione pubblica delle particolarità culturali e identitarie degli individui - non fornisce delle risposte normativamente soddisfacenti per affrontare questo tipo di situazione. Per affronta- 
re politicamente le discriminazioni culturali, occorre «vedere», riconoscere, $\mathrm{i}$ gruppi in questione, e determinare delle modalità politiche che non si riferiscano a dei cittadini neutri, epurati delle loro affiliazioni e lealtà culturali, ma soprattutto a dei cittadini differenziati, situati in contesti specifici, membri di gruppi aventi caratteristiche culturali e sociali particolari.

Questi due approcci coinvolgono due concezioni del riconoscimento pubblico diverse. Il modello liberale classico si basa essenzialmente sull'idea di riconoscimento dell'eguaglianza come principio d'integrazione; il secondo modello si fonda sul riconoscimento della differenza come mezzo per raggiungere una migliore integrazione. Cercherò ora di mostrare, brevemente, gli elementi salienti di queste due concezioni del riconoscimento.

\section{Riconoscimento dell'uguaglianza: accesso alla comunità politica}

Riconoscendo ai non-membri la possibilità di diventare membri della comunità, lo Stato stabilisce chi, come e per quale motivo ha accesso al territorio nazionale. Attaverso queste modalità, lo Stato controlla sia la grandezza e la composizione interna della comunità politica (nel caso delle naturalizzazioni o delle leggi d'attribuzione della nazionalità) che quelle della società civile (nel caso di stranieri residenti, temporanei o permanenti, o di rifugiati politici). Il modello dei «tre cerchi» del governo svizzero, gli accordi di Schengen o la legge sull'asilo fanno parte di questa forma di riconoscimento.

Fondamentalmente, la domanda che è alla base di questo approccio è: che cosa attribuisce a un individuo il diritto di diventare cittadino di uno Stato? Detto in un'altra maniera: quali caratteristiche devono essere riconosciute in un individuo affinché costui possa diventare un membro della comunità? Esistono varie risposte normative a questa domanda. Esse si situano su un asse che va dalle posizioni nazionaliste (Scruton 1990) ${ }^{6}$ alla teoria delle «frontiere aperte» (Carens 1995), secondo la quale lo stato liberale non ha il diritto di precludere agli stranieri l'accesso al suo territorio. In mezzo, le posizioni moderate

6 Per il quale «il vero prezzo della comunità [...] è l'inviolabilità, l'intolleranza, l'esclusione e la convinzione che il senso della vita dipende dall'obbedienza ma anche dalla diffidenza verso il nemico» $(1990,310)$. 
(liberali e comunitarie) secondo le quali la comunità ha il diritto inviolabile di decidere chi sono i suoi membri (Walzer 1987). Tutte queste teorie affrontano il problema del multiculturalismo essenzialmente come un problema d'accesso dei potenzialmente «diversi» a una comunità (nazionale e politica). Il problema dell'integrazione sociale e politica delle minoranze culturali è relativamente secondario, perché il presupposto di partenza è che l'integrazione dei nuovi membri si opererà attraverso la cittadinanza, unico collante in grado di unire questo universo composito. Sono i diritti individuali di partecipare alla determinazione della volontà collettiva, di libertà d'espressione, di lavorare e di beneficiare del welfare che permetteranno ai nuovi venuti d'integrarsi. In sostanza, dunque,

la cittadinanza crea [...] una comunità sottoposta alla legge; coloro che vi appartengono fanno parte del sistema di regole che li protegge l'uno dall'altro e, creando una sorta di club, dagli estranei (Dahrendorf 1974, 672).

Il riconoscimento dell'uguaglianza e della protezione giuridica incarnate dalla cittadinanza permetterà ai nuovi venuti d'integrarsi anche socialmente. Questo perché l'attribuzione della cittadinanza non implica solo l'ottenimento di uno statuto giuridico e politico, ma anche l'accesso alla comunità nazionale. Quindi, l'attribuzione della cittadinanza esprime il riconoscimento statale dell'integrabilità dei nuovi membri. In questo senso, l'integrazione, per essere legittima, deve avere una componente culturale, quella dell'identità collettiva incarnata dall'appartenenza nazionale (D. Miller 1993). Il modello assimilazionista esprime con forza questo legame.

Questo modello è molto contestato, non solo nel mondo nord-americano, ma anche in Europa. L'ethos identitario prodotto dalla filosofia esistenzialista, i mutamenti culturali degli anni ' 60 , i processi di globalizzazione e le ondate migratorie, rendono questo modello sempre meno pertinente da un punto di vista sociologico e politico. Quest'insieme di caratteristiche strutturali fa sì che sempre più individui sentano la necessità di identificarsi

con una data sub-cultura che [li] istituisca nella [loro] differenza: sia essa etnica, culturale, di sesso, d'orientamento sessuale o, perché no, d'età. Qualsiasi di tali differenze individuali socialmente rilevanti può servire da catalizzatore per una rivendicazione di questo tipo (Roman 1995, 152).

Riprendendo la definizione di cittadinanza proposta da Van 
Gunsteren $(1988,732)$ - ossia: «la cittadinanza è una risposta alla domanda 'Chi sono?' e 'Cosa devo fare?' quando essa viene posta nella sfera pubblica» - si può dire che nelle società multiculturali le possibilità che degli individui rispondano «sono principalmente un appartenente al gruppo x e, accessoriamente, un cittadino» sono molto più alte che in un sistema a forte integrazione socio-nazionale. Il problema è cruciale, visto che, come afferma Zincone $(1992,60)$, si tratta di sapere se

la frattura tra nazione e cittadinanza, il fatto che i cittadini non siano più uniti da legami di lingua, di tradizioni, e di cultura non mini i fondamenti etici di quella solidarietà spontanea che sentiamo verso il gruppo cui pensiamo di appartenere naturalmente, una solidarietà indispensabile per operare quei gravosi trasferimenti di reddito tra membri e componenti del gruppo che sono necessari a produrre diritti sociali. Il particolarismo delle regioni, delle comunità etniche e religiose si rivela già fin d'ora come un potente nemico di quello stato sociale che si prefiggeva diritti di base uguali per tutti i cittadini.

\section{Il riconoscimento della differenza: integrazione nella cittadinanza}

La riflessione concernente il riconoscimento d'integrazione intende affrontare normativamente la questione della fuga dalla cittadinanza come principio dell'identità politica (e della determinazione della volontà collettiva). Essa rappresenta una critica radicale dell'ottimismo nelle virtù integrative della concezione liberale. Il presupposto di base è che l'attribuzione di diritti individuali non sia sufficiente a garantire l'integrazione di membri collocati diversamente rispetto alla distribuzione delle risorse simboliche, materiali e cognitive in una data comunità. La cittadinanza rappresenta una parte importante di queste risorse. Essendo una risorsa rara, essa è inegualmente distribuita. Quindi, lungi dall'essere un fattore d'unione, tende a costituire un fattore di divisione in seno alle società democratiche. Questa premessa contraddice l'analisi classica di Marshall (1976), secondo la quale le conquiste della cittadinanza (l'ottenimento di diritti civili, politici e sociali) permetterebbero di ridurre gli effetti delle ineguaglianze di classe. Ė sempre più chiaro, in effetti, che

le modernizzazioni democratiche non possono raffigurarsi come semplici passaggi dal «fuori» al «dentro», come un lento ma continuo processo di inclusione di categorie prima escluse. La fascia del cosiddetto «fuori» è continuamente ricostituita: da fenomeni di emarginazione interna (i nuovi poveri di diritti), da fenomeni di immigrazione esterna (i nuovi non cittadini), da fenomeni di inclusione imperfetta o incompiuta (i vecchi poveri di diritti). E gli «in- 
terni» della cittadinanza, la configurazione e l'architettura dei diritti muta ad ogni nuovo ingresso o ad ogni nuova resistenza o espulsione (Zincone 1992, 9-10).

Sono quindi queste categorie di esclusi o «poveri in diritti» che si tratta di integrare (o re-integrare) nella comunità politica. Se il riconoscimento del primo tipo concerne il chi dei non membri ha il diritto di diventare membro, ossia la possibilità di avere dei diritti, il riconoscimento del secondo tipo porta sulle modalità politiche che dovrebbero permettere a questi attori di beneficiare di diritti effettivi. Si tratta, in sostanza, di sapere come integrare i formalmente integrati (i cittadini) e i non integrati (non membri).

Se il riconoscimento «1» implica una concezione individuale dell'integrazione, la riflessione sul riconoscimento «2» si focalizza prioritariamente sulla dimensione collettiva dei gruppi di esclusi. Gli individui non sono considerati come atomi sparsi che si tratta d'integrare singolarmente, ma come appartenenti a delle minoranze culturali. E precisamente quest'appartenenza (volontaria o socialmente attribuita) che, spesso, determina la loro mancanza d'integrazione. Fondamentalmente, dunque, alla base di questo approccio risiede l'intuizione aristotelica secondo la quale l'uguaglianza democratica implica il fatto di trattare gli uguali in modo uguale $\mathrm{e} i$ differenti in modo differente (Young 1990).

Come per il riconoscimento «1», esistono varie maniere d'impostare analiticamente il problema. Per taluni, certe forme di riconoscimento pubblico temporanee dovrebbero permettere di contrastare le forme di discriminazione strutturale endemica (pensiamo alle quote di rappresentazione delle donne o delle minoranze etniche, o ancora all'affirmative action); per altri, il riconoscimento pubblico delle differenze culturali rappresenta un diritto che deriva dai presupposti normativi stessi del liberalismo (Kymlicka 1995a). In quest'ottica, la cultura ${ }^{7}$ costituisce un «bene primario» (Rawls 1993) che è necessario salvaguardare (dunque, proteggere attraverso dei diritti) affinché gli indivi-

7 Kymlicka (1995a, 76) utilizza il concetto di «societal culture», ossia una «cultura che attribuisce un senso ai comportamenti/modi di essere dei propri membri per tutte le loro attività svolte nel campo sociale, educativo, religioso, economico e durante il tempo libero, sia nell'ambito pubblico che privato. Queste culture tendono alla concentrazione territoriale e si basano su una lingua comune». Kymlicka propone quindi una definizione abbastanza restrittiva della cultura. 
dui possano fare delle scelte di vita autonome e libere. Esiste anche una corrente analitica che rivendica l'importanza del riconoscimento delle differenze culturali ma che, allo stesso tempo, è critica nei confronti dei diritti liberali. Si tratta dell'approccio dei teorici detti postmoderni. Anche se mi sembra sterile dal punto di vista normativo, questo paradigma generale merita considerazione, perché contribuisce sensibilmente a chiarire la natura dei problemi di esclusione e marginalizzazione in seno alla cittadinanza. Oltretutto, i suoi presupposti teorici ispirano considerabilmente la «identity politics», l'espressione politica più genuina della critica alla cittadinanza liberale.

Secondo questa interpretazione, l'universalismo liberale si basa, di fatto, sulla creazione e l'esclusione dei gruppi culturali minoritari. Il liberalismo, inteso come insieme di valori e pratiche politiche, riproduce la relazione schmittiana fra amico e nemico, o fra le identità maggioritarie - considerate normali - e quelle minoritarie, viste come anormali o patologiche. In questo contesto, il principio di neutralità delle istituzioni non è nient'altro che uno specchio per le allodole. L'uguaglianza giuridica non esprime la realizzazione di un principio morale universalista, ma soprattutto i rapporti di potere che caratterizzano, a un dato momento, una società particolare. Il liberalismo chiede ai cittadini di essere neutrali nei confronti di strutture di potere che non lo sono, ma che avvantaggiano certe identità a scapito di altre. Si tratta, in sostanza, di un particolarismo mascherato da universalismo. Per questi autori, oltretutto, i diritti universali non permettono l'espressione dell'autenticità delle varie identità culturali. Col loro effetto omogeneizzante, inibiscono la fluidità della soggettività individuale. Le comunità liberali sono formate da soggetti che incarnano delle identità e delle differenze incommensurabili, eminentemente conflittuali, impossibili da fissare in un sistema stabile di relazioni (Honig 1994; Mouffe 1994). Identità e differenza intrattengono un rapporto simbiotico, inscindibile. La cittadinanza, intesa come principio identitario della comunità politica, non fa eccezione: stabilisce $i$ suoi «altri», determinando chi, non disponendo di questo statuto o non vivendo secondo i suoi dettami, è meno virtuoso o meno razionale, quindi «diverso». L'esclusione è quindi operata sulla base delle caratteristiche individuali o collettive degli attori, della loro presunta diversità o incompatibilità con i valori espressi dal sistema democratico. Una cittadinanza autentica si esprime nella lotta contro ogni tentativo d'imposizione di categorie uni- 
versali a scapito d'identità particolari (per esempio, il cittadino a scapito della donna, dell'omosessuale, del credente).

E facile capire come, portata alle sue estreme conseguenze, questa logica comporta la negazione della cittadinanza come principio identitario comune:

se non vi è alcun limite al pluralismo culturale, allora ci si avvicina, evidentemente, al punto in cui la stessa nozione di cittadinanza in quanto realtà esistenziale si dissolve nel nulla (Beiner 1995, 8).

L'«esistenzialismo politico», associato alla rivendicazione del riconoscimento della propria autenticità, implica virtualmente l'impossibilità di creare una comunità di cittadini che condividano qualcosa di più di un semplice statuto formale. Nella migliore delle ipotesi, può dare origine a una serie di comunità indipendenti, ognuna basata su un referente identitario che gli attori sociali, a un certo momento della loro storia, considerano come non-negoziabile, come costitutivo della loro autentica soggettività. L'appartenenza alla comunità (e l'adesione all'ethos che la caratterizza) sono infatti percepite come il solo criterio dell'autenticità individuale. Visto che non si può negoziare la propria autenticità, la possibilità di stabilire delle comunità fondate su di un consenso sulle regole e su certi contenuti minimi condivisi (presupposti della solidarietà e responsabilità collettive) diventa un'operazione difficile.

Come ho detto, questa corrente teorica mi sembra incapace di definire una posizione normativa chiara in materia di riconoscimento, cittadinanza e integrazione. Quest'ultima, ad esempio, è simbioticamente legata al conflitto. Se i diritti hanno effettivamente una dimensione alienante, com'è possibile giustificare i diritti di riconoscimento? Al di là di questa contraddizione, comunque, le analisi menzionate in precedenza illustrano delle tendenze sociologiche pertinenti. La storia dei vari regimi liberali è ricca di esempi che mostrano come l'universalismo si sia realizzato attraverso delle pratiche discriminatrici nei confronti di certe minoranze (pensiamo alla strategia d'inferiorizzazione dei colonizzati illustrata da Franz Fanon, o all'esclusione di minoranze su basi razziali, etniche o sessuali). Ma, detto questo, occorre fare un passo avanti, ossia cercare di definire il significato normativo del riconoscimento nel quadro delle società multiculturali. Per far questo, mi sembra utile insistere su un aspetto comune alle pratiche di esclusione su base culturale: si tratta della negazione del rispetto delle minoranze culturali e 
dei loro membri (che sono identificati col gruppo anche se non ne fanno parte), fatto che toglie alla cittadinanza il suo potenziale integratore.

\section{Riconoscimento e produzione politica del rispetto}

Se intesa come il principio di riconoscimento intersoggettivo alla base della comunità politica, la teoria della cittadinanza deve assumere, da parte dei membri, una simmetria di disposizioni. Come dice Ferry $(1991,163)$,

in quanto cittadini, i co-societari non sono designati dai predicati di qualità che permettono d'altronde di assegnarli ad appartenenze particolari: si autoistituiscono tramite la relazione che dichiarano di avere.

Affinché possa funzionare, questa relazione deve essere basata sulla disposizione al rispetto reciproco. Rispetto, in questo senso, significa essere considerato, malgrado le differenze cognitive e materiali, degno e atto a partecipare alla determinazione dei valori collettivi. Non è quindi in un'accezione morale che considero il rispetto, ma come una disposizione psicologica affinché un rapporto civile possa istituirsi fra gli attori sociali e politici. È una caratteristica essenziale del regime democratico aver introdotto e generalizzato la politica dell'uguale dignità, quella che permette ai soggetti di emanciparsi dalla loro subordinazione nei confronti di costruzioni simboliche e politiche che ne limitano l'autonomia di scelta e la libertà di perseguire le loro concezioni del bene. Il rispetto, in sostanza, deriva dall'autonomia individuale, dal fatto di essere considerato atto a assumere le proprie scelte morali e politiche e di essere in grado di condividere delle esperienze comuni ${ }^{8}$. Ora, in una società multiculturale, fortemente frammentata, composta da attori che hanno delle concezioni diverse e incommensurabili del bene, il rispetto costituisce una risorsa sociale rara, distribuita in modo inegualitario. Come afferma giustamente S.M. Miller $(1993,48)$,

la capacità che una collettività ha di rispettare se stessa dipende in gran parte dal rispetto degli altri - il modo in cui il gruppo viene considerato e trattato, l'esperienza avuta nelle interazioni, il carattere più o meno accettabile di certi modelli per la società e le prospettive economiche e sociali del gruppo.

8 «La qualità morale dell'agente svanisce se questi non è riconosciuto come soggetto di scelte libere» (Galeotti 1994, 55). 
L'aspetto saliente della mia argomentazione è che una cittadinanza non basata sul rispetto reciproco perde il suo carattere democratico. La categoria normativa del rispetto mi sembra quindi rappresentare un aspetto importante per poter concepire un nuovo modello d'integrazione multiculturale. Dunque, deve essere integrata nella teoria della cittadinanza. Concordo con Taylor $(1993,41-42)$ quando scrive che

la nostra identità è plasmata, in parte dal riconoscimento o dal mancato riconoscimento o, spesso, da un misconoscimento da parte di altre persone, per cui un individuo o un gruppo può subire un danno reale, una reale distorsione, se le persone o la società che lo circondano gli rimandono, come uno specchio, un'immagine di sé che lo limita o sminuisce o umilia. Il non riconoscimento o misconoscimento può danneggiare, può essere una forma di oppressione che imprigiona una persona in un modo di vivere falso, distorto e impoverito.

Esiste quindi un nesso fra riconoscimento e rispetto. Nel quadro delle società postmoderne, nelle quali l'identità non è più un fatto acquisito e giustificato metafisicamente o oggettivamente, ma il frutto di una costante costruzione sociale, il rispetto può emanare solo dalla visibilità, dal riconoscimento intersoggettivo fra gli individui e i gruppi. Quindi, se, da una parte, il rispetto può essere prodotto socialmente, dall'altra può anche essere socialmente negato. In questo caso, il rispetto si trasfor$\mathrm{ma}$ in una risorsa politica. In effetti, il rispetto collettivo apre le porte dell'autonomia individuale, mentre la mancanza di rispetto contribuisce a tenerle chiuse. E ciò malgrado il fatto che gli individui possiedano la cittadinanza'.

La mancanza di riconoscimento, che implica la condizione d'invisibilità sociale e politica, costituisce un'arma efficace di negazione del rispetto ${ }^{10}$. Ma anche i processi di riconoscimento pubblico negativo, fondati su politiche di esclusione su basi razziali, etniche, religiose o di genere, implicano una negazione radicale del rispetto e, ovviamente, dei diritti di cittadinanza. Mi sembra quindi importante sottolineare che la natura degli atti

9 Ad esempio, secondo Steele «il fatto di essere nero negli Stati Uniti significa che la propria stima dell'individuo verrà colpita più spesso. La tendenza a dubitare di se stesso che nasce da queste ferite verrà rafforzata dalla reputazione di inferiorità della razza nera» $(1990,43)$.

10 Come sostiene Esman «il diniego di riconoscimento è un'arma nelle mani degli avversari etnici [...] Una lotta per il riconoscimento ed il rispetto collettivo ha grandi possibilità di sorgere quando una minoranza etnica crede che la propria condizione è stata calpestata dalle autorità» $(1994,218)$. 
istituzionali influenza sensibilmente le relazioni fra gli attori nella società civile. In altri termini, certe politiche pubbliche sono più idonee alla produzione collettiva del rispetto che altre. Il problema è chiaro: il fatto che lo Stato dichiari o dimostri con le sue politiche di non apprezzare certe categorie di attori culturali (che siano cittadini o meno) autorizza implicitamente la società civile a trattarli con poco rispetto, quindi non nell'ottica di un'integrazione ma secondo dinamiche di esclusione (Zincone 1994). Detto con altre parole, più le categorie deboli saranno private di dignità e di rispetto, più saranno l'oggetto di violenza razzista o sessista da parte della cultura dominante. Non voglio affermare un rapporto di causa a effetto, ma è plausibile pensare che esista un legame stretto fra rispetto politico e rispetto socioculturale.

La negazione del rispetto crea, alla lunga, un rafforzamento dell'identità delle categorie discriminate. Ricorrendo alla terminologia di Anderson, si può dire che la narrazione della discriminazione produce delle comunità immaginarie, ma ciò non toglie che esse determinino degli effetti politici reali. Un modello di cittadinanza che non considera questa dimensione costituisce, nel migliore dei casi, una mera categoria giuridica, incapace di promuovere una integrazione interculturale. Ma nel peggiore dei casi, invece di essere uno spazio di creazione del consenso e di definizione delle regole democratiche, diventa il luogo della battaglia fra attori politici che rivendicano delle identità non negoziabili, per i quali l'orizzonte politico e morale è definito dalla comunità d'appartenenza. Queste ultime non sono costruite necessariamente sulla base di un referente oggettivo (l'etnia, la razza, la religione, il sesso), ma su delle percezioni soggettive dei rapporti con i gruppi dominanti. Insomma: la comunità non è solamente «quello che è», ma è soprattutto «quello che sento e che provo» soggettivamente. Ancora una volta, una tale dinamica implica virtualmente la tribalizzazione dello spazio pubblico, con la conseguenza che la democrazia rappresentativa diventa l'equivalente di una «scatola vuota» (Zolo 1992).

A mio modo di vedere, quindi, la ricerca di modalità politiche e sociali di creazione e di mantenimento del rispetto rappresenta un punto nodale per stabilire dei processi d'integrazione efficaci e legittimi nelle democrazie multiculturali. Detto in altre parole, la distribuzione del rispetto rappresenta una questione politica centrale per concepire delle forme migliori d'integrazione nel quadro delle democrazie multiculturali. Non illudiamoci: 
non si può creare integrazione o rispetto solamente «per decreto», attraverso l'attribuzione di diritti da parte dello Stato. Il riconoscimento pubblico delle differenze e le politiche del rispetto non rappresentano quindi una panacea. Occorre definire delle politiche pubbliche mirate a risolvere problemi specifici in contesti specifici. Ma la comprensione della natura del problema può permettere la creazione di un principio ispiratore delle politiche atte a migliorare l'efficacia dell'integrazione. Senza forme di riconoscimento pubblico delle differenze, la sola via possibile verso l'integrazione è quella assimilazionista. Ma ciò sembra difficilmente compatibile con i presupposti sociologici e antropologici delle società liberali postmoderne. Considerare seriamente il riconoscimento come modello d'integrazione implica la definizione di regole che sappiano gestire la tensione fra le rivendicazioni d'integrazione su basi universalistiche («noi siamo come voi, malgrado il fatto che abbiamo delle pratiche culturali differenti») e le rivendicazioni d'integrazione su basi particolaristiche («noi non siamo autentici, quindi liberi, se non possiamo mantenere e vedere riconosciuta la nostra specificità culturale»). In un tale contesto, imporre l'accettazione di un principio d'identità sostanziale (quindi potenzialmente esclusivo, come la Nazione, la Comunità linguistica o religiosa) non sembra essere una via politicamente fruttuosa e perseguibile.

Il modello della cittadinanza differenziata ha come scopo di cercare di limitare l'impatto delle tendenze centrifughe proprie alla comunità dei cittadini (e dei futuri cittadini). Per far questo, ritiene che, oltre ai diritti individuali, l'individuo deve beneficiare di un surplus di diritti che gli sono attribuiti in funzione della sua appartenenza a una minoranza culturale. Ma un punto deve essere chiaro: contrariamente a quello che propone Taylor (1993), questi diritti non devono essere finalizzati a garantire la sopravvivenza intergenerazionale di una forma culturale, ma a proteggere e rinforzare l'integrazione politica e sociale di individui e categorie endemicamente escluse o marginalizzate politicamente. In altri termini, il riconoscimento collettivo delle caratteristiche del gruppo dovrebbe aiutare gli individui a recuperare un rispetto politico e delle risorse giuridiche atte a permettergli di cercare di modificare le cause della propria discriminazione. Ma è anche importante sottolineare che l'attribuzione di diritti culturali non deve negare i diritti individuali liberali. I diritti culturali sono una risorsa in più per i membri di gruppi discriminati, non il mezzo per creare delle «comunità 
culturali» che hanno diritti collettivi più importanti di quelli dei membri che le compongono.

Galeotti $(1994,162)$ esprime chiaramente la relazione fra riconoscimento, rispetto e integrazione che ritengo essere alla base dell'idea di cittadinanza differenziata:

La domanda del riconoscimento pubblico delle differenze intende proprio capovolgere la ratio dell'inclusione individuale: se la strategia dell'inclusione individuale non produce di fatto l'inclusione del gruppo nel suo insieme, e se ciò che rende l'inclusione individuale un sogno impossibile è la disapprovazione, il sospetto, il disprezzo attribuito all'identità diversa, spesso condannata all'invisibilità pubblica, allora solo il riconoscimento di questa identità, purificata però dei suoi significati sociali negativi, può costituire la premessa perché gli individui della minoranza si sentano pubblicamente accettati e rispettati per ciò che globalmente sono.

A dire il vero, le democrazie occidentali prevedono già un largo campionario di diritti di espressione della differenza. Pensiamo, ad esempio, alla possibilità per i Sikh di non mettere il casco in Inghilterra, al diritto d'eccezione degli Amish di frequentare la scuola pubblica negli Stati Uniti, o al recente spostamento delle votazioni dalla domenica al lunedì per permettere agli ebrei italiani di poter votare rispettando i loro principi religiosi. Oltretutto, in parecchi paesi europei e in certi cantoni svizzeri, gli stranieri beneficiano di diritti di voto a livello locale. Ciò malgrado, queste concessioni mi sembrano emanare più dall'applicazione del principio di tolleranza che non dall'idea che è necessario promuovere una migliore integrazione dei gruppi, e che il riconoscimento di parte delle loro differenze culturali è un modo per farlo.

La dimensione simbolica della politica del rispetto è considerevole. I diritti di riconoscimento, se non accompagnati da un discoso pubblico mirato a spiegare l'esigenza della produzione del rispetto politico (e non morale), possono originare più marginalizzazione di quella che sono supposti combattere. Il caso dell'affirmative action negli Stati Uniti è significativo. Benché sia una politica pubblica che ha positivamente contribuito a rendere visibile il problema della discriminazione razziale, la sua applicazione ha dato luogo a dei risultati discutibili. In effetti, un individuo impiegato in un'industria unicamente in virtù di un criterio razziale (e non di competenza) godrà difficilmente del rispetto dei suoi colleghi. Ciò origina una situazione paradossale: al miglioramento della situazione economica individuale (possibilità di avere un lavoro), corrisponde un aumento della 
stigmatizzazione della comunità alla quale l'individuo appartiene, considerata una comunità di assistiti, incapace di realizzare l'American Dream senza l'aiuto dello Stato. Come detto in precedenza, in una tale situazione, il pericolo per il sistema democratico è che, di fronte alla stigmatizzazione costante, l'individuo percepisca il suo gruppo culturale d'appartenenza come solo e unico luogo di riconoscimento e ottenimento del rispetto individuale e collettivo. Questo comporta una fuga dalla cittadinanza, quindi dal politico inteso come ricerca del bene comune.

\section{Conclusioni}

Come ho avuto modo di affermare più volte, la politica del riconoscimento, finalizzata a una migliore integrazione, comporta dei cambiamenti sostanziali della concezione liberale della cittadinanza. Il più significativo risiede nel passaggio da un modello d'integrazione basato sui diritti individuali a un modello nel quale i diritti collettivi occupano uno spazio importante. Più precisamente, occorre concepire delle modalità d'integrazione dei gruppi minoritari che non neghino completamente le loro appartenenze e identità di gruppo e quindi definire un modello di cittadinanza democratica che consideri anche (ma non solo) le differenze culturali. In questo senso, la politica del riconoscimento non implica un rivoluzionamento delle pratiche democratiche, ma un'altra maniera per cercare di realizzare meglio l'ideale democratico. Il fatto di considerare il rispetto come una risorsa inegualmente distribuita e di mettere l'accento sulla necessità di una produzione politica del rispetto, significa definire un insieme di principi che devono ispirare le politiche pubbliche. Per fare questo, occorre che le istituzioni indichino quali gruppi devono essere oggetto di un riconoscimento. Non possono quindi essere neutre e cieche alle specificità culturali come il modello liberale richiede. È questo il senso della cittadinanza differenziata: a partire da una unità complessa, composta da membri aventi delle affiliazioni conflittuali, si tratta di stabilire delle modalità politiche che non neghino, ma neanche capitolino di fronte all'espressione delle identità culturali (Phillips 1993).

$\mathrm{Ma}$ è anche importante ribadire che, come ogni categoria normativa, il riconoscimento non costituisce il rimedio ai problemi dell'integrazione. Il fatto di considerarlo seriamente non 
implica che occorra riconoscere pubblicamente tutti i gruppi che lo richiedono. Bisogna stabilire dei criteri politici di determinazione di chi ha diritto al riconoscimento. Per far questo, bisognerà fare in modo che tutti gli attori (anche quelli nei confronti dei quali si applicherà il riconoscimento) possano partecipare alla determinazione collettiva di questi criteri. Questo è un fattore importante, perché se è vero che la politica dell'identità rappresenta il vettore privilegiato che i vari gruppi adottano per produrre dell'autorispetto, si pone anche il problema della legittimità democratica di questi gruppi. Sulla base di caratteristiche generali come la razza, l'etnia, la lingua o la religione, certi leader politici si proclamano i rappresentanti di queste comunità immaginarie, ma senza mai essere stati eletti dai membri della comunità stessa. Questo esempio mostra come l'istituzionalizzazione di forme di riconoscimento «2» non costituisce la panacea per tutti i mali. Sicuramente darà origine a degli effetti perversi. Ma questo non mi sembra un argomento sufficiente per abbandonare il progetto. Non si rimette in causa il principio dei diritti umani solamente perché la maggioranza degli Stati non li applica. Al contrario, occorre raddoppiare le forze affinché siano sempre più riconosciuti e applicati. Lo stesso dicasi per i diritti di riconoscimento d'integrazione. Nelle società liberali è necessario rivitalizzare la cittadinanza come principio d'integrazione. Ma non si può farlo negando il problema, affermando che non è politico e che quindi altri meccanismi (il mercato, il lavoro, la famiglia) devono risolverlo. Queste istituzioni sono importanti, ma non possono funzionare senza una volontà politica che le sostenga e le legittimi.

Le ineguaglianze economiche sono un rivelatore potente delle tensioni multiculturali, e la politica del riconoscimento non costituisce di certo un'alternativa al welfare, ma un contributo ulteriore. È chiaro che questo tipo di politica porrà il problema dell'accettazione da parte dei gruppi sociali che non ne beneficiano, soprattutto in un periodo di crisi economica e di trasformazioni strutturali importanti. Non mi sembra però che si possa affermare che la trasposizione politica dei principi liberali non si sia urtata con gli stessi problemi. Non è la forza dei diversi che minaccia l'ordine democratico, ma la loro debolezza. Una maniera per rinforzare il peso politico di questi gruppi consiste nel riconoscimento istituzionale dell'importanza delle rivendicazioni identitarie. La speranza è che, grazie a ciò, la cittadinanza recupererà parte della sua funzione d'integrazione $e$ di costruzione intersoggettiva delle pratiche democratiche. 


\section{Riferimenti bibliografici}

Barber, B. (1995), Blood Brothers, Consumers or Citizens? Three Models of Identity-Ethnic, Commercial and Civic, testo della conferenza presentata al Centre d'études ethniques, Montreal.

Beiner, R. (a cura di) (1995), Theorizing Citizenship, Albany, State University New York Press.

Carens, J. (1995), Aliens and Citizens: The Case for Open Borders, in Beiner (1995), pp. 229-253.

Connolly, W. (1991), Identity/Difference. Democratic Negotiations of Political Paradox, Ithaca, Cornell University Press.

Dahrendorf, R. (1974), Citizenship and Beyond: The Social Dynamics of an Idea, in «Social Research», vol. 41, pp. 673-701.

Esman, M. (1994), Etbnic Politics, Ithaca, Cornell University Press.

Fanon, F. (1961), Les damnés de la terre, Paris, F. Maspero.

Ferry, J.M. (1991), Les puissances de l'expérience. Tome 2, Paris, Cerf.

Galeotti, A.E. (1994), La tolleranza. Una proposta pluralista, Napoli, Liguori Editore.

Gellner, E. (1989), Nations et nationalisme, Paris, Payot.

Gianni, M. (1995), Multiculturalisme et Démocratie: quelques implications pour la théorie de la citoyenneté, in «Revue Suisse de Science Politique», vol. I, pp. 3-39.

Gitlin, T. (1995), The Twilight of Common Dreams, New York, Metropolitan Books.

Gutmann, A. (1993), Introduzione, in Taylor (1993), pp. 11-39.

Honig, N. (1994), Difference, Dilemmas, and the Politics of Home, in «Social Research», vol. 61, pp. 563-597.

Kymlicka, W. (1995a), Multicultural Citizenship, Oxford, Oxford University Press.

- (a cura di) (1995b), The Rights of Minority Cultures, Oxford, Oxford University Press.

Lukes, S. (1995), Toleration and the Realities of Symbolic Politics, relazione presentata al colloquio «On Toleration» dell'UNESCO, Siena, aprile.

Marshall, T.H. (1976), Cittadinanza e classe sociale, Torino, UTET.

Miller, D. (1993), In Defense of Nationality, in «Journal of Applied Philosophy», vol. 10, pp. 3-16.

Miller, S.M. (1993), The politics of Respect, in «Social Policy», vol. 23, pp. 44-51.

Mouffe, C. (1994), Le politique et ses enjeux, Paris, La Découverte.

Phillips, A. (1993), Democracy and Difference, Cambridge, Polity Press.

Pizzorno, A. (1993), Le radici della politica assoluta, Milano, Feltrinelli. Rawls, J. (1993), Political Liberalism, New York, Columbia University Press. 
Raz, J. (1994), Multiculturalism, in «Dissent», winter, pp. 67-79.

Roman, J. (1995), Un multiculturalisme à la française?, in «Esprit», 212, pp. $145-160$.

Scruton, R. (1990), In Defense of the Nation, in R. Scruton, The Philospher on Dover Beach, Manchester, Carcanet, pp. 299-337.

Steele, S. (1990), The Content of our Character, New York, St. Martin's Press.

Taylor, C. (a cura di) (1993), Multiculturalismo. La politica del riconoscimento, Milano, Anabasi.

Van Gunsteren, H. (1988), Admission to Citizenship, in «Ethics», 98, pp. 731-741.

Walzer, M. (1987), Sfere di giustizia, Milano, Feltrinelli.

- (1992), Che cosa significa essere americani, Venezia, Marsilio.

Young, I.M. (1990), Justice and the Politics of Difference, Princeton, Princeton University Press.

Zincone, G. (1992), Da sudditi a cittadini, Bologna, Il Mulino.

- (1994), Uno schermo contro il razzismo, Roma, Donzelli.

Zolo, D. (1992), Il principato democratico, Milano, Feltrinelli. 